\title{
A GENERALISATION OF MINKOWSKI'S SECOND INEQUALITY IN THE GEOMETRY OF NUMBERS
}

\author{
A. C. WOODS
}

(Received 24 June 1965)

Let $K$ be a bounded open convex set in euclidean $n$-space $R_{n}$ symmetric in the origin 0 . Further let $L$ be a discrete point set in $R_{n}$ containing 0 and at least $n$ linearly independent points of $R_{n}$. Put $m_{i}=\inf u_{i}$ extended over all positive real numbers $u_{i}$ for which $u_{i} K$ contains $i$ linearly independent points of $L, i=1,2, \cdots, n$.

Denote by $P$ a parallelopiped in $R_{n}$ centred at 0 . For each positive integer $N$ denote by $L(N P)$ the number of points of $L$ contained in $N P$. Put

$$
d(L, P)=\lim \inf (V(N P) / L(N P)), \quad N \rightarrow \infty,
$$

where $V(N P)$ denotes the Jordan content of $N P$ and

$$
d(L)=\inf d(L, P)
$$

extended over all nondegenerate parallelopipeds $P$. It is assumed here that $L$ is such that $d(L)$ is finite and positive. In particular if $L$ is a lattice then $d(L)$ is the determinant of $L$ and in this case Minkowski's second inequality in the geometry of numbers asserts that

$$
m_{1} m_{2} \cdots m_{n} V(K) \leqq 2^{n} d(L) .
$$

The object here is to show that (1) remains true if the restriction of $L$ to a lattice is replaced by the weaker condition

(A) if $X \in L$ and $Y \in L$ then either $X-Y$ or $Y-X$ is in $L$. Examples of such sets are obtained by taking a lattice $\Lambda$, a positive integer $m$ and a fixed point $X$ of $R_{n}$ such that the sets

$$
A, A+X, A+2 X, \cdots, A+m X
$$

are pairwise disjoint. With $L$ as the union of these $m+1$ sets it is evident that $L$ satisfies $(A)$ and that $d(L)=d(\Lambda) /(m+1)$. In particular with $m=1$, $L$ becomes the familiar double lattice relating homogeneous and inhomogeneous problems in the geometry of numbers and (1) becomes a trans- 
ference theorem. M. Bleicher has pointed out in correspondence that there are sets $L$ other than those given here with property $A$.

Minkowski's original proof of (1) for lattices has been simplified by Weyl [3] and Cassels [4], and a quite different proof has been given by Davenport [1]. Further simplifications of both lines of proof are given in Bambah, Woods and Zassenhaus [5]. It is the version by Bambah of Davenports proof that is used here to obtain the generalisation stated above and it is interesting to note that the Minkowski method of proof appears to break down for this wider class of sets.

\section{Proof of the generalisation}

THEOREM 1. Suppose that $S$ is a bounded set in $R_{n}$. Let $\chi(X)$ be its characteristic function. Suppose further that

$$
I=\int_{-\infty}^{\infty} \cdots \int_{-\infty}^{\infty} \chi(X) d x_{1} \cdots d x_{n}
$$

exists and $I>d(L)$. Then the sets $S+A, A \in L$ overlap so that there exists points $X, Y$ in $S$ such that $X-Y \in L$.

Proof. By way of contradiction assume that the sets $S+A, A \in L$ do not overlap. Let $P$ denote the cube given by

$$
\max \left(\left|x_{1}\right|, \cdots,\left|x_{n}\right|\right) \leqq 1 .
$$

Since $S$ is bounded there exists a positive integer $k$ such that $S$ is contained in $k P$. For a fixed positive integer $N$ consider the set $Z$ of points $A$ of $L$ that lie in the cube NP. Define

$$
F(X)=\sum_{A \in Z} \chi(X-A)
$$

By hypothesis the sets $S+A$ do not overlap, hence $F(X) \leqq 1$ for all $X$. Further $F(X)=0$ if $X$ is not in the cube $(N+k) P$. Therefore

$$
\begin{aligned}
(2 N+2 k)^{m} \geqq & \int_{-\infty}^{\infty} \cdots \int_{-\infty}^{\infty} F(X) d x_{1} \cdots d x_{n} \\
& =\sum_{A \in Z} \int_{-\infty}^{\infty} \cdots \int_{-\infty}^{\infty} \chi(X-A) d x_{1} \cdots d x_{n} \\
& =\sum_{A \in Z} \int_{-\infty}^{\infty} \cdots \int_{-\infty}^{\infty} \chi(X) d x_{1} \cdots d x_{n} \\
& =L(N P) I .
\end{aligned}
$$

Thus

$$
\left.I \leqq(V(N P) / L(N P))((2 N+2 k) / 2 N)^{n}\right)
$$


Let $N$ tend to infinity; this implies that

$$
I \leqq d(L, P) \text { and therefore also } I \leqq d(L),
$$

the contradiction that proves the theorem.

Lemma 1. If $T$ is a nonsingular transformation of $R_{n}$ into itself then $d(T(L))=\|T\| d(L)$.

Proof. Let $P$ be a parallelopiped centred at 0 . For a fixed positive integer $N$ the number of points of $T(L)$ in $N P$ is the same as the number of points of $L$ in $N T^{-1}(P)$. Hence

$$
\begin{aligned}
d(T(L), P) & =\lim \inf \left[V(N P) / L\left(N T^{-1}(P)\right)\right] \\
& =\|T\| \lim \inf \left[V\left(N T^{-1}(P)\right) / L\left(N T^{-1}(P)\right)\right] \\
& =\|T\| d\left(L, T^{-1}(P)\right) .
\end{aligned}
$$

Thus $d(T(L))=\inf _{P}\|T\| d\left(L, T^{-1}(P)\right)=\|T\| d(L)$, which proves the lemma.

Lemma 2. Let $n_{1}, n_{2}, \cdots, n_{n}$ be $n$ positive real numbers such that

$$
n_{1} \leqq n_{2} \leqq \cdots \leqq n_{n}
$$

There exist sets $K_{1}, K_{2}, \cdots, K_{n}$ such that

(i) $K_{1}=\frac{1}{2} n_{1} K$,

(ii) $K_{i} \subset \frac{1}{2} n_{i} K$ for $i=1,2, \cdots, n$,

(iii) If $X, Y \in K_{i}$ for $i>1$ and $x_{i}=y_{i}, \cdots, x_{n}=y_{n}$ then there exist points $X^{\prime}, Y^{\prime}$ in $K_{i-1}$ such that $X-Y=X^{\prime}-Y^{\prime}$,

(iv) If $\chi_{i}(X)$ is the characteristic function of $K_{i}$ then

$$
\begin{aligned}
V_{i} & =\int_{-\infty}^{\infty} \cdots \int_{-\infty}^{\infty} \chi_{i}(X) d x_{1} \cdots d x_{n} \\
V_{i} & =\left\{\begin{array}{lll}
\left(\frac{1}{2} n_{1}\right)^{n} V(K) & \text { if } i=1 \\
\left(n_{i} / n_{i-1}\right)^{n-i+1} V_{i-1} & \text { if } i>1 .
\end{array}\right.
\end{aligned}
$$

exists and

A proof of this lemma is given in [5].

TheOREM 2. If $L$ has property $(A)$ then (1) holds.

Proof. Since $L$ is discrete and contains $n$ linearly independent points of $R_{n}$, it follows that there exist $n$ linearly independent points $F_{1}, F_{2}, \cdots$, $F_{n}$ of $L$ such that $F_{i}$ lies on the boundary of $m_{i} K$ for each $i=1,2, \cdots, n$. From lemma 1 it follows that $(1)$ is an invariant inequality under nonsingular linear transformations of $R_{n}$, so without loss of generality it may be assumed that the coordinate system is such that $F_{i}$ has coordinates of the form $\left(f_{1}, f_{2}, \cdots, f_{i}, 0, \cdots, 0\right)$ for each $i=1,2, \cdots, n$. Now if $\frac{1}{2} m_{i} K$ contains 
two points $X=\left(x_{1}, \cdots, x_{n}\right)$ and $Y=\left(y_{1}, \cdots, y_{n}\right)$ such that $X-Y$ is in $L$ then, by the convexity and symmetry of $K, X-Y$ is in $m_{i} K$ and so cannot be linearly independent of $F_{1}, \cdots, F_{i-1}$; hence $x_{i}=y_{i}, \cdots$, $x_{n}=y_{n}$. Apply lemma 2 with $n_{i}=m_{i}$ for $i=1,2, \cdots, n$ to obtain the sets $K_{1}, \cdots, K_{n}$. Assume by way of contradiction that (1) is false so that

Then

$$
m_{1} \cdots m_{n} V(K)>2^{n} d(L) .
$$

$$
V_{n}=m_{1} \cdots m_{n}\left(\frac{1}{2}\right)^{n} V(K)>d(L)
$$

and by theorem 1 there exist points $X, Y$ in $K_{n}$ such that $0 \neq X-Y \in L$. Since $K_{n} \subset \frac{1}{2} m_{n} K$ this implies that $x_{n}=y_{n}$, and by property (1) of the sets $K_{i}, K_{n-1}$ contains points $X^{(1)}, Y^{(1)}$ such that

$$
X-Y=X^{(1)}-Y^{(1)} \text {. }
$$

Since $K_{n-1} \subset \frac{1}{2} m_{n-1} K$ this implies that $x_{n-1}^{(1)}=y_{n-1}^{(1)}, x_{n}^{(1)}=y_{n}^{(1)}$ and there exist points $X^{(2)}, Y^{(2)}$ in $K_{n-2}$ with

$$
X-Y=X^{(2)}-Y^{(2)} .
$$

Repeating this argument a number of times we obtain points $X^{*}, Y^{*}$ in $\frac{1}{2} m_{1} K$ such that $X-Y=X^{*}-Y^{*}$. But $X^{*}-Y^{*} \in L$ implies that $X^{*}=Y^{*}$ and $X-Y=0$, which is a contradiction. This proves the theorem.

\section{A comment on Minkowski's method}

Minkowski's method relies upon the fact that the measure of any measurable subset of $R_{n}$ in the quotient space of $R_{n}$ modulo a lattice of dimension $\leqq n$ is monotone, that is to say, if $C<C^{\prime}$ then the measure of $C$ does not exceed the measure of $C^{\prime}$ in the quotient space. Such a measure can be generalised to sets $L$ other than lattices as follows. Let $C$ be a measurable subset of $R_{n}$. If $C$ is bounded there exist at most a finite number of points $Z_{1}, \cdots, Z_{k}$ other than 0 such that $C+Z_{i}$ intersects $C$. Denote by $M_{i+1}$ the measure of the set of points $X$ of $C$ such that $C$ lies in exactly $i$ of the sets $C+Z_{1}, \cdots, C+Z_{k}$ and define

$$
M(C)=M_{1}+\frac{1}{2} M_{2}+\frac{1}{3} M_{3}+\cdots \frac{1}{k} M_{k} .
$$

Now if $M(C)$ is monotone so that $C \subset C^{\prime}$ implies $M(C) \leqq M\left(C^{\prime}\right)$ for bounded measurable sets $C, C^{\prime}$ then it is possible to show that (1) holds provided $d(L)$ is replaced by the upper bound of $M(C)$ taken over all measurable bounded sets $C$. However the author has been unable to find any interesting sets $L$ other than lattices that have this property. 


\section{References}

[1] H. Davenport; "Minkowski's inequality for the minimum associated with a convex body", Quart. J. Math. (Oxford) 10 (1939), 199-121.

[2] H. Minkowski, Geometrie der Zahlen (Teubner 1896), Chapter 5.

[3] H. Weyl, "On geometry of numbers", Proc. Lond. Math. Soc. (2) 47 (1942), 268-289.

[4] J. W. S. Cassels, An introduction to the geometry of numbers (Springer, 1959).

[5] R. P. Bambah, A. C. Woods and H. Zassenhaus, "Three proofs of Minkowski's second inequality in the geometry of numbers", J. Aust. Math. Soc. 5 (1965), 453-462.

The Ohio State University

Columbus, Ohio 